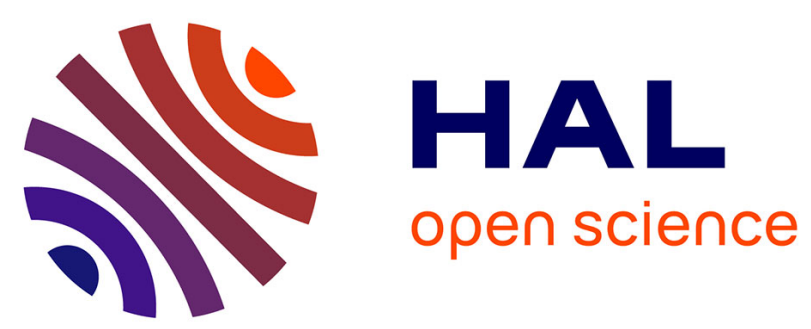

\title{
Chemokine-biased robust self-organizing polarization of migrating cells in vivo
}

\author{
Adan Olguin-Olguin, Anne Aalto, Benoît Maugis, Aleix Boquet-Pujadas, \\ Dennis Hoffmann, Laura Ermlich, Timo Betz, Nir S. Gov, Michal \\ Reichman-Fried, Erez Raz
}

\section{To cite this version:}

Adan Olguin-Olguin, Anne Aalto, Benoît Maugis, Aleix Boquet-Pujadas, Dennis Hoffmann, et al.. Chemokine-biased robust self-organizing polarization of migrating cells in vivo. Proceedings of the National Academy of Sciences of the United States of America, 2021, 118 (7), pp.e2018480118. 10.1073/pnas.2018480118 . hal-03298487

\section{HAL Id: hal-03298487 \\ https://hal.sorbonne-universite.fr/hal-03298487}

Submitted on 23 Jul 2021

HAL is a multi-disciplinary open access archive for the deposit and dissemination of scientific research documents, whether they are published or not. The documents may come from teaching and research institutions in France or abroad, or from public or private research centers.
L'archive ouverte pluridisciplinaire HAL, est destinée au dépôt et à la diffusion de documents scientifiques de niveau recherche, publiés ou non, émanant des établissements d'enseignement et de recherche français ou étrangers, des laboratoires publics ou privés.

\section{(1) $(1) \Theta$}

Distributed under a Creative Commons Attribution - NonCommercial - NoDerivatives| 4.0 


\title{
Chemokine-biased robust self-organizing polarization of migrating cells in vivo
}

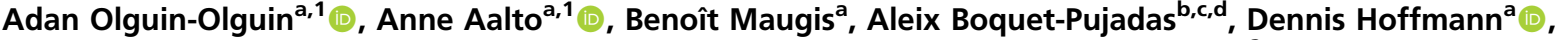

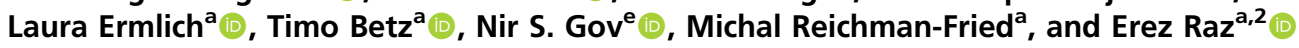

${ }^{a}$ Institute of Cell Biology, Center for Molecular Biology of Inflammation, University of Münster, 48149 Münster, Germany; ${ }^{b}$ Bioimage Analysis Unit, Institut Pasteur, 75105 Paris, France; 'CNRS, UMR 3691, 75015 Paris, France; ' Sorbonne Université, 75005 Paris, France; and ${ }^{\mathrm{e}}$ Department of Chemical and Biological Physics, Weizmann Institute of Science, 76100 Rehovot, Israel

Edited by Peter N. Devreotes, Johns Hopkins University School of Medicine, Baltimore, MD, and approved January 5, 2021 (received for review September 13, 2020)

To study the mechanisms controlling front-rear polarity in migrating cells, we used zebrafish primordial germ cells (PGCs) as an in vivo model. We find that polarity of bleb-driven migrating cells can be initiated at the cell front, as manifested by actin accumulation at the future leading edge and myosin-dependent retrograde actin flow toward the other side of the cell. In such cases, the definition of the cell front, from which bleb-inhibiting proteins such as Ezrin are depleted, precedes the establishment of the cell rear, where those proteins accumulate. Conversely, following cell division, the accumulation of Ezrin at the cleavage plane is the first sign for cell polarity and this aspect of the cell becomes the cell back. Together, the antagonistic interactions between the cell front and back lead to a robust polarization of the cell. Furthermore, we show that chemokine signaling can bias the establishment of the front-rear axis of the cell, thereby guiding the migrating cells toward sites of higher levels of the attractant. We compare these results to a theoretical model according to which a critical value of actin treadmilling flow can initiate a positive feedback loop that leads to the generation of the front-rear axis and to stable cell polarization. Together, our in vivo findings and the mathematical model, provide an explanation for the observed nonoriented migration of primordial germ cells in the absence of the guidance cue, as well as for the directed migration toward the region where the gonad develops.

amoeboid migration | bleb | cell polarity | chemotaxis | ezrin

E tablishing and maintaining polarity is essential for a range of different cellular functions (1). This requirement is particularly critical for single-cell migration, a process that depends on the definition of a front-rear axis, which, in turn, dictates the direction of movement. This important issue has previously been investigated primarily in cells such as Dictyostelium discoideum, leukocytes, fibroblasts, and neural crest cells (2-7), cell types that employ actin polymerization to generate physical force to advance their leading edge in a form of lamellipodia. These studies suggest that polarity is generated and stabilized by processes of local positive feedback control loops, long-range negative feedback loops, and by control of protrusion formation through membrane tension, thereby facilitating forward movement of the cells (8-11).

In contrast to actin-powered lamellipodia-based migration, a different cell migration strategy exhibited by diverse cell types in physiological contexts, as well as in pathological conditions is characterized by the generation of bleb-type protrusions at the cell front (12-14). Blebs are spherical in shape and are powered by hydrostatic pressure and cytoplasmic flow (15-17). Here, a net forward movement is achieved via polarized bleb formation driven by inflow of cytoplasm and concomitant retraction of the opposite side of the cell. Several molecular processes have been found to contribute to the generation of blebs. Most notably, actomyosin contractility is required to generate the intracellular hydrostatic pressure and to induce breaks in the cell cortex that promote the separation of the plasma membrane from the underlying actin filaments (18). This process is therefore controlled by the balance between contractility, membrane tension, and membrane-cortex attachment (15-17, 19). Nevertheless, little is known about the mechanisms responsible for defining the front of blebbing cells and establishing the rear. Similarly, the nature of the interactions between the front and the rear is not well understood in this context. A theoretical model that describes these interactions is the universal coupling of speed and cell persistence (UCSP) model (20). An important component in this model for establishing cell polarization is the net actin retrograde flow away from the side of the cell that becomes the cell front. According to this model, the front can be maintained at this location by a positive feedback between the actin flow and the flow of inhibitors of actin polymerization away from it (21). Experimentally, in the context of cell migration, actin retrograde flow was first shown to be important in processes of focaladhesion-dependent motility. The flow results from a combination of actin polymerization against the cell membrane at the leading edge and nonmuscle myosin II activity (22-24).

An important model for studying bleb-driven motility in an in vivo context is that of zebrafish primordial germ cells (PGCs) (25). Zebrafish PGCs migrate within the embryo employing blebs and are directed by the chemokine Cxcl12a and its receptor Cxcr4b (26). When guided by the chemokine as well as when migrating in random directions in its absence, PGCs alternate

\section{Significance}

Bleb-driven cell migration plays important roles in diverse biological processes. Here, we present the mechanism for polarity establishment and maintenance in blebbing cells in vivo. We show that actin polymerization defines the leading edge, the position where blebs form. We show that the cell front can direct the formation of the rear by facilitating retrograde flow of proteins that limit the generation of blebs at the opposite aspect of the cell. Conversely, localization of bleb-inhibiting proteins at one aspect of the cell results in the establishment of the cell front at the opposite side. These antagonistic interactions result in robust polarity that can be initiated in a random direction, or oriented by a chemokine gradient.

Author contributions: A.O.-O., A.A., B.M., N.S.G., M.R.-F., and E.R. designed research; A.O.-O., A.A., B.M., D.H., L.E., and N.S.G. performed research; A.B.-P., T.B., and N.S.G. contributed new reagents/analytic tools; A.O.-O., A.A., and B.M. analyzed data; and A.O.-O., A.A., and E.R. wrote the paper.

The authors declare no competing interest.

This article is a PNAS Direct Submission.

This open access article is distributed under Creative Commons Attribution-NonCommercialNoDerivatives License 4.0 (CC BY-NC-ND).

${ }^{1}$ A.O.-O. and A.A. contributed equally to this work.

${ }^{2}$ To whom correspondence may be addressed. Email: erez.raz@uni-muenster.de.

This article contains supporting information online at https://www.pnas.org/lookup/suppl/ doi:10.1073/pnas.2018480118/-/DCSupplemental.

Published February 11, 2021. 
between two distinct modes of behavior namely "run" and "tumbling" (27). During run phases, PGCs extend blebs in the direction of movement and actively migrate, while during the tumbling phases the cells are morphologically apolar, form blebs in all directions, and are immotile (27). This periodic sequence of events where PGCs lose and regain polarity makes these cells an excellent model for studying the establishment and maintenance of cell polarity of blebbing cells and for determining the role of chemokine signaling in this process.

Determining the precise course of events leading to cell motility and the interdependency of different events that contribute to the polarization of migrating cells in vivo is challenging. Nevertheless, by studying PGC migration within early zebrafish embryos we could describe the molecular cascade leading to cell polarization. We found that polarization is initiated by a biased actin polymerization at one aspect of the cell. This initial symmetry-breaking event determines and orients the front-rear axis of the cell by directing streaming of specific proteins to the future back. Proteins such as Ezrin and extended synaptotagminlike $2 \mathrm{a}$ that are transported to the cell back can inhibit the formation of blebs, thereby specifying the rear of the cell. At the same time, the transport of these proteins away from the putative cell front makes blebbing more likely to occur there. In addition, we provide evidence suggesting that blebbing enhances actin polymerization at the front, thereby establishing a positive feedback with the retrograde actin flow. The combination of the positive and negative feedback reinforces the initial cell polarization event to establish a persistent polarity. Furthermore, while this cascade can be initiated at random positions around the cell perimeter, the primary polarization event can be biased by chemokine signals, thereby directing cell migration toward specific regions in the embryo where the attractant is expressed. Last, our experimental observations could be described by the set of equations based on the UCSP theoretical model. This model predicts that persistent cell polarization can be established above a critical level of actin polymerization-induced treadmilling, which breaks the cell symmetry by advection of bleb-inhibiting proteins to the other aspect of the cell, thereby maintaining the polarized actin flow.

\section{Results}

A Polarized Distribution of Molecules and Structures along the Front-Rear Axis of Migrating PGCs. To elucidate the events and cross-interactions among cellular elements that facilitate cell polarization, we first set out to identify proteins and structures that are asymmetrically distributed within migrating PGCs. The front of migrating PGCs exhibits blebs and contains a dense F-actin structure (termed actin brushes) (Fig. $1 \mathrm{~A}$; see also ref. 28). These actin structures are similar to those found in pseudopods and in some cases, they appear to be capable of pushing the membrane forward. However, during PGC migration, they mainly serve as a platform from which blebs emerge at the cell front. Specifically, we observed that $16 \%$ of the front protrusions are pseudopod-like structures while $84 \%$ are clear blebs (SI Appendix, Fig. S1 $A$ and $B$ ). A detailed analysis of the distribution of blebs around the cell perimeter revealed that they preferentially form in the direction of migration, with fewer and smaller blebs at the cell rear (SI Appendix, Fig. S1 $C$ and D). Next, to identify additional elements that are distributed unevenly along the front-rear axis of the polarized cell, we determined the relative position of organelles and selected molecules. Interestingly, while the endoplasmic reticulum (ER) is distributed throughout the cell body, it is absent from expanding blebs at the cell front (Fig. 1B). Two other structures, the microtubule organizing center (MTOC, visualized by labeling the MTs) and the Golgi apparatus (Fig. $1 C$ and $D$, respectively) reside at the rear of the cell, behind the nucleus. Importantly, Ezrin, a member of the ERM family that links the plasma membrane to the actin cortex, is strongly enriched at the rear of the cell (Fig. $1 E$ ), similar to findings in certain other
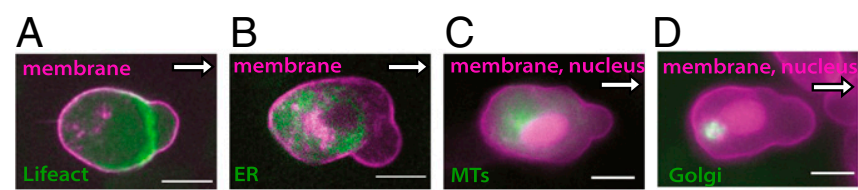

$\mathrm{E}$

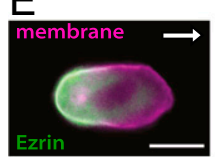

F

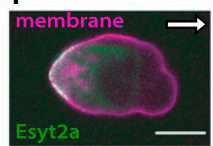

G

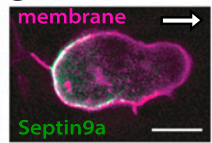

H
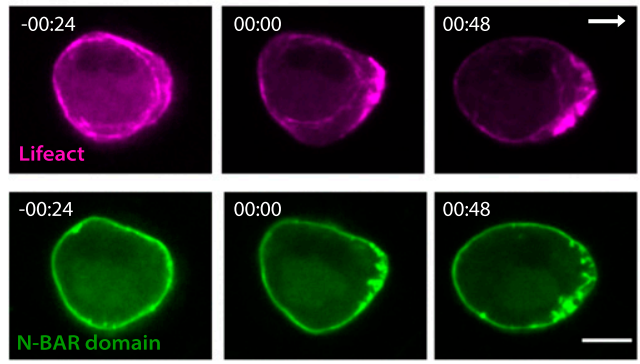

I
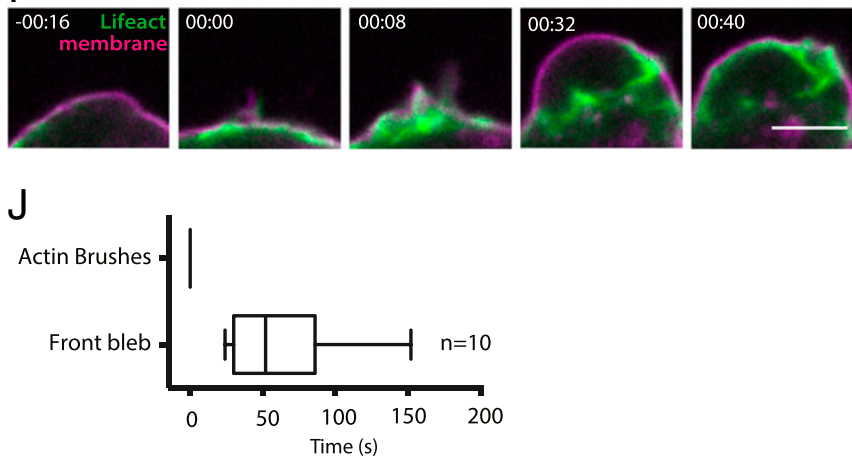

Fig. 1. Polarized distribution of proteins and subcellular structures within wild-type migrating PGCs and steps of self-organized ligand-independent polarized bleb formation. $(A-G)$ The localization fluorescent protein fusions (green) labeling $(A)$ actin (Lifeact), $(B)$ the ER (calreticulin signal peptide), $(C)$ the MTs (Clip-170H protein), $(D)$ the Golgi (N terminus of beta 1,4galactosyltransferase fusion), $(E)$ Ezrin, $(F)$ extended synaptotagmin-like $2 \mathrm{a}$ (Esyt2a) and (G) Septin9a, relative to the plasma membrane (labeled by a farnesylation signal from c-Ha-Ras fused to fluorescent protein [magenta]) and the nucleus (magenta in $C$ and $D$ ). White arrows indicate the direction of migration. (Scale bars, $10 \mu \mathrm{m}$.) $(H)$ The formation of membrane invaginations labeled with the N-BAR domain (human Amphiphysin fused to YFP [green]), at the new front where Lifeact-labeled actin accumulates (magenta). Times are in minutes and seconds. (Scale bar, $10 \mu \mathrm{m}$.) (I) The formation of actin brushes at the new front and inflation of the first bleb. Times are in minutes and seconds. (Scale bar, $5 \mu \mathrm{m}$.) $(J)$ The time of bleb formation relative to the time of establishment of actin brushes at the cell front (median, 5 and 95 percentiles are indicated. $n=$ number of cells, time is in seconds).

cell types $(29,30)$. Interestingly, extended synaptotagmin-like 2a (Esyt2a), which belongs to a family of proteins that connect the ER to the plasma membrane $(31,32)$ is also localized to the rear of the cell (Fig. $1 F)$. Last, we found that Septin9a is enriched at the cell rear (Fig. $1 G$ ), where it could act in a complex controlling cortical rigidity (reviewed in ref. 33). Taken together, we detected actin accumulation at the cell front where blebs form and determined the localization of several molecules that could potentially limit the formation of blebs at the rear of the cell.

The Definition of the Cell Front and the Formation of the Rear during Exit from Tumbling in Ligand-Independent PGC Polarization. While the precise role of actin brushes at the cell front in bleb formation 
is not completely understood, we made two observations relevant to this issue. First, consistent with the fact that myosin light chain kinase (MLCK) is enriched at the front of migrating PGCs (28), we found it localized within the actin brushes (SI Appendix, Fig. $\mathrm{S} 2 A)$. This distribution is independent of myosin contractility as observed upon expression of dominant negative Rho kinase (DN-ROCK) (SI Appendix, Fig. S2B). It is conceivable therefore, that actin brushes could bias blebbing to the front by the recruitment of MLCK, thereby enhancing contractility at this part of the cell. Conversely, blebbing activity is not essential for the initial formation of actin brushes, as brushes can form in cells in which contractility is reduced by DN-ROCK expression. Similarly, actin polymerization is observed in cells in which membrane release for bleb formation is inhibited by overexpression of the N-BAR domain of Amphiphysin (SI Appendix, Fig. S2C). A second potential mechanism by which the brushes could increase blebbing concerns their influence on the structure of the membrane around the cell perimeter which can affect bleb formation (34). While membrane invaginations were previously shown to be enriched at the leading edge of migrating PGCs (35), here we show that these invaginations develop as soon as actin brushes form (Fig. $1 H$ ). Importantly, while blebbing is not required for the initial formation of actin brushes (SI Appendix, Fig. S2C), blebbing is important for maintaining actin polymerization. This is because the persistence of the actin brushes in nonblebbing cells is lower (SI Appendix, Fig. S2D), indicating that the retrograde flow is repressed in cells in which blebbing is reduced.

As a first step in exploring the mechanisms responsible for the establishment of polarity in the migrating cells, we determined the temporal course of events during polarity acquisition in the absence of the Cxcl12 guidance cue. Toward this end, we took advantage of the fact that cells establish new polarity upon exiting the tumbling phase and entering the run phase.

Blebs differ from actin-powered lamellipodia regarding their dynamics, molecular composition, and morphology. Interestingly, however, we found that similar to findings in mesenchymal cells (36), polymerized actin at the future cell front is the earliest (defined as "time 0") polarized structure in PGCs that are about to exit tumbling (Figs. $1 I$ and $J$ and $2 A-D$ ). This event is followed by the formation of a bleb within a minute after accumulation of actin at the front (Fig. $1 I$ and $J$ ), with Ezrin, Esyt2a, and Septin9a becoming localized to the cell back about 2 min after the appearance of the actin brushes (Fig. $2 A, B$, and $D, S I$ Appendix, Fig. S3D and Movie S1). We quantified the polarization of these proteins as performed in a recent study (37), using an approach considering angular distribution of the fluorescent signal with respect to the center of mass of the cell and the center of mass of the fluorescent signal (Materials and Methods). This quantification shows that following the initial formation of actin brushes at the new front (time 0 ) an increase in the polarity index of the back markers is observed (Fig. $2 A^{\prime}-B^{\prime}$ and SI Appendix, Fig. S3 $A, B, D^{\prime}$, and $D^{\prime \prime}$, arrows point at the center of the fluorescent signal). Interestingly, the time of positioning of the MTOC at the cell rear is highly variable and often represents a late event in ligand-independent PGC polarization (Fig. $2 C$ and $C^{\prime}$ and SI Appendix, Fig. S3C). Thus, while in migrating cells the MTOC is found at the rear of the cell (Fig. $1 C$ ), its positioning is unlikely to be instructive during the initial establishment of the front-rear axis. Together, these observations are consistent with the idea that the site at which the front forms, determines the position of the cell rear that is characterized by localization of proteins such as Ezrin, Esyt2a, and Septin9a.

Collectively, these results show a temporal connection between actin brushes formation and polarized blebbing. The formation of brushes is subsequently followed by the establishment of the full front-rear axis as specific proteins become localized to the cell back.
The Rear of the Cell Is Defined Prior to the Cell Front following Cell Division in Ligand-Independent PGC Polarization. Another key stage at which PGCs become polarized is at the end of cell division. During mitosis, the cells assume a round morphology and as judged by the distribution of the different molecular markers, the cells are apolar (Fig. $2 E-G$, time point 00:00). Interestingly, the cleavage furrow that develops during the last stages of mitosis harbors proteins that we found to reside at the cell rear (e.g., Ezrin) (38). Accordingly, Ezrin and Esyt2a are the first proteins to become localized along the front-rear axis upon repolarization (Fig. $2 E$ and $F$ ). Under these conditions, in the absence of the chemokine, actin brushes and the future cell front form away from the part of the cell marked by Ezrin and Esyt2a (Fig. $2 E$ and $F$ and Movie S2). As mitosis progresses, the MTOC that is located initially at the future cell front gradually translocates toward the cell back (Fig. $2 G$ time point 23:44, Movie S2). These results suggest that while the cell front initiates polarization when PGCs exit from tumbling (Fig. $2 A-C$ ), in the particular case of cell division in the absence of chemokine signaling, the rear of the cell can also direct cell polarization and thereby determine the location of the front (Fig. $2 E$ and $F$ ).

The Role of Rac1 in Orienting the Front-Rear Axis in Blebbing Cells. Since we found that as PGCs exit from tumbling the front is established before the rear, we sought to directly assess the effect of actin polymerization on the process. To this end, we first studied the dynamics of Rac1 activation in the absence of the chemoattractant. To follow active Rac1 protein distribution, we monitored the distribution of an active Rac1-associated protein, PAK-1 (39). Specifically, we examined the localization of the GTPase binding domain (GBD) of PAK-1 in PGCs as they acquire polarity. Interestingly, we observed the formation of a PAK-1 crescent (Fig. $3 A$, time point $-00: 16$ ) prior to the polarized formation of the actin brushes at that location (Fig. $3 A$, time point 00:00), which as presented above (Fig. $2 A-D$ ), precedes the accumulation of Ezrin, Esyt2a, and Septin9a at the cell back. Importantly, the PAK-1 GBD recruitment to the membrane of newly formed blebs (Fig. $3 A$, time point 00:16 PAK-1 GBD) and the subsequent formation of actin brushes at the new leading edge help to maintain the front-biased actin accumulation (Fig. 3A, time point 00:24 Lifeact). To examine whether formation of the actin-rich structures at the front by Rac1 activation is instructive and sufficient for the definition of the cell rear, we employed a photoactivatable version of Rac1 (40) (Fig. $3 B$ and Movie S3). We found that indeed, activation of Rac1 in tumbling cells directed the formation of the front to the site of activation (Fig. 3B). Remarkably, activating Rac1 at the rear of already-polarized cells led to the formation of two actinrich areas at the same time (Fig. $3 B^{\prime}$ ). Subsequently, the area that exhibited a stronger actin signal was further maintained, followed by a newly forming rear at the opposite side of the cell, as reflected by Ezrin enrichment at that position (Fig. $3 B^{\prime}$ ). These results thus show that the formation of the cell front is sufficient for dictating where the rear will form, and that the front arises from a global competition through the actin flow that transports linker proteins across the cell length.

To further investigate the role of Rac1 in the polarization of PGCs, we inhibited its activity by expressing a dominant negative (DN) form of the protein (Rac1T17N) (41). As a result, the formation of the actin brushes at the cell front was inhibited. Additionally, as we have previously shown, DN-Rac1 expressing cells are not elongated, do not migrate, and exhibit apolar blebbing activity [Fig. 3C and Movie S4 (42)]. This phenotype could result from the weakening of the actomyosin cortex coupled with residual contractility, which leads to more frequent separation of the plasma membrane from the cortex and to bleb formation. Indeed, a lower density of cortex-membrane binding should result in a lower nucleation barrier for bleb initiation, 


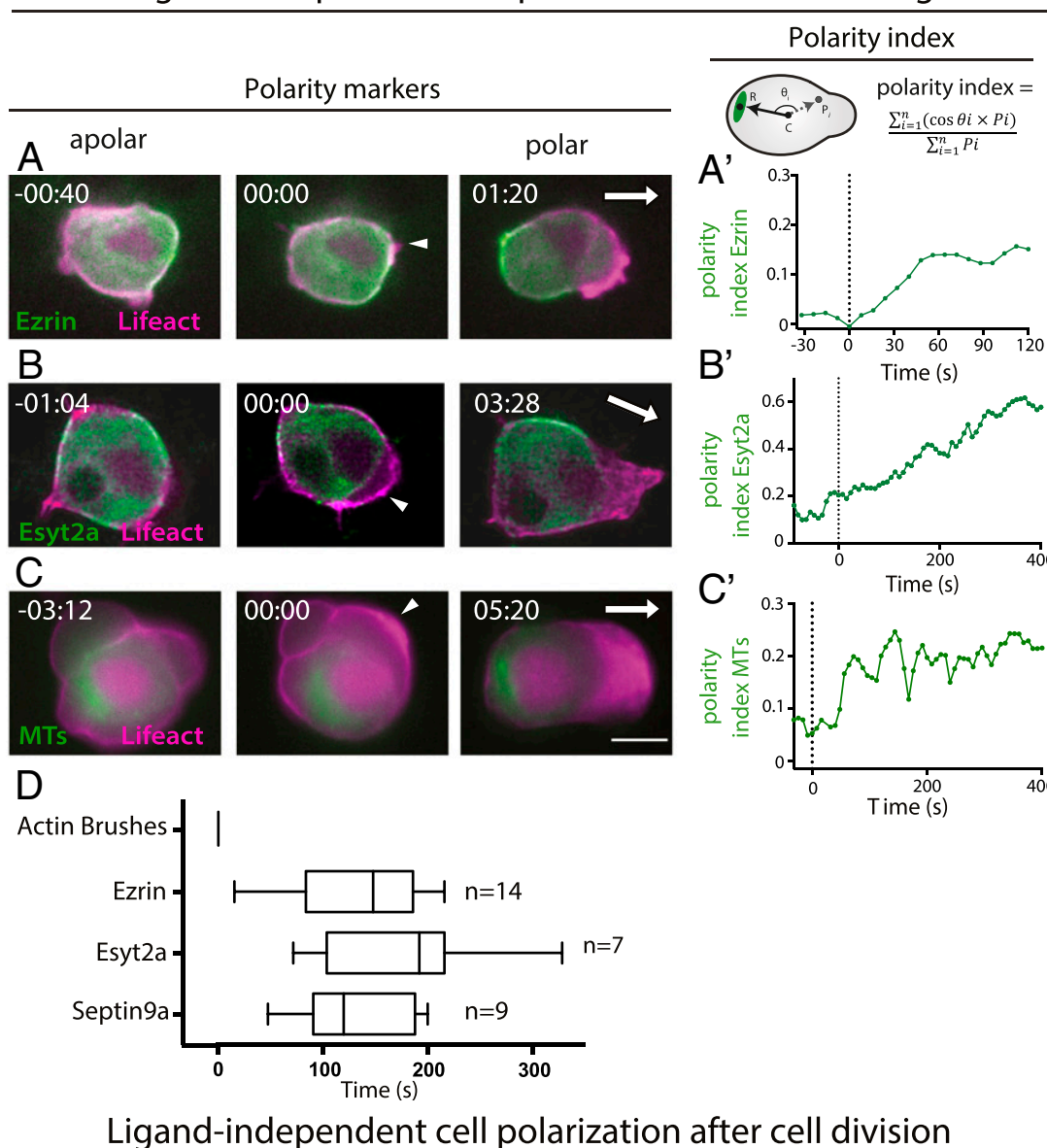

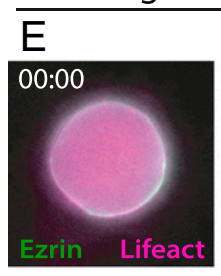
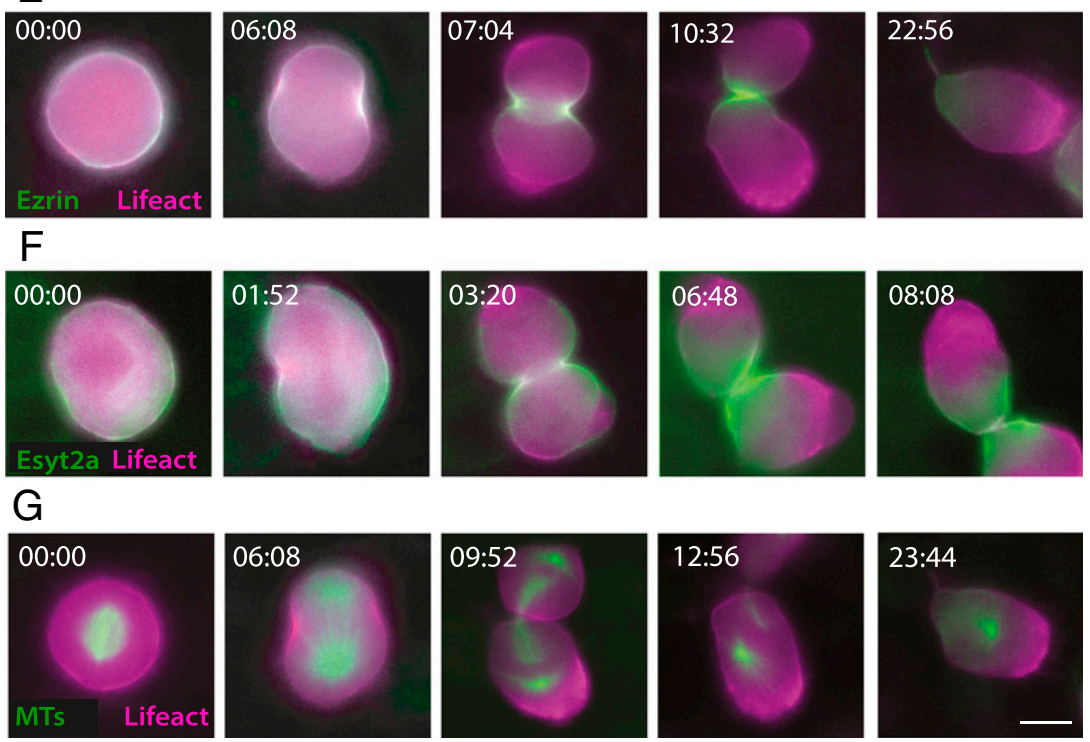

Fig. 2. Steps of ligand independent self-organizing cell polarization. $(A-C)$ The establishment of the cell rear in the absence of receptor signaling as determined by the distribution of $(A)$ Ezrin, $(B)$ Esyt2a, and $(C)$ MTs (Clip170H) in green. The cell front is marked by Lifeact (magenta). Apolar tumbling cells (Left), the appearance of actin within the new cell front (time 00:00, Middle), and the localization of the proteins at the rear of the cell (Right) is presented. Times indicate minutes and seconds, before and after the establishment of the front and the white arrows point at the direction of migration. The arrowheads indicate the actin brushes. (Scale bar, $10 \mu \mathrm{m}$.) $\left(A^{\prime}-C^{\prime}\right)$ Time course of the polarity index establishment of the polarization of Ezrin $\left(A^{\prime}\right)$ Esyt2a $\left(B^{\prime}\right)$, or MTs $\left(C^{\prime}\right)$ within the cells presented in $A-C$. In $A^{\prime}$ and $B^{\prime}$ the signal was measured only at the level of the membrane; time appears in seconds. (D) Time of ligand-independent polarization of the rear markers relative to the time of actin accumulation at the cell front (time 0 ) (median, 5 and 95 percentiles are presented, $n=$ number of cells, time is in seconds). ( $E-G)$ Establishment of polarity following cell division. Lifeact presented in magenta and the rear markers in green: $(E)$ Ezrin, $(F)$ Esyt2a, and $(G)$ MTs. Apolar cells prior to cytokinesis (Left) and subsequent panels present the progression through cell division, with the polarized daughter cell (Right). (Scale bar, $10 \mu \mathrm{m}$.) leading to more frequent blebs (19). Under these conditions, two of the examined molecular markers for the rear of the polarized cell (Ezrin, MTOC) were not localized to a specific location within the cell (Fig. $3 C$ and Movie S4). The polarity of Ezrin and MTOC was quantified using the same software as in Fig. $2 A^{\prime}-C^{\prime}$, revealing a low and fluctuating polarity index (Fig. $3 C$ ). Together, these results show that Rac activity is essential for generation of a cell front and as such, it is subsequently needed for the establishment of the rear.

In addition to the role of Rac1 activation in establishing the cell front, we found that the formation of the actin brushes plays an additional role in the polarization of the cell. Since the ER is excluded from blebs at the cell front (Figs. $1 B$ and $3 D^{\prime}$ ), it is possible that the actin brushes found ahead of the ER constitute 
A Rac activity during ligand-independent cell polarization
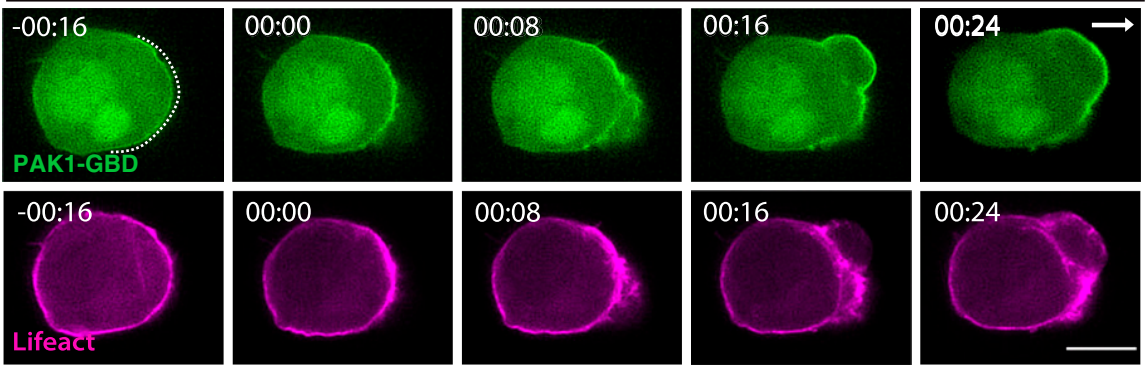

Photoactivatable-Rac
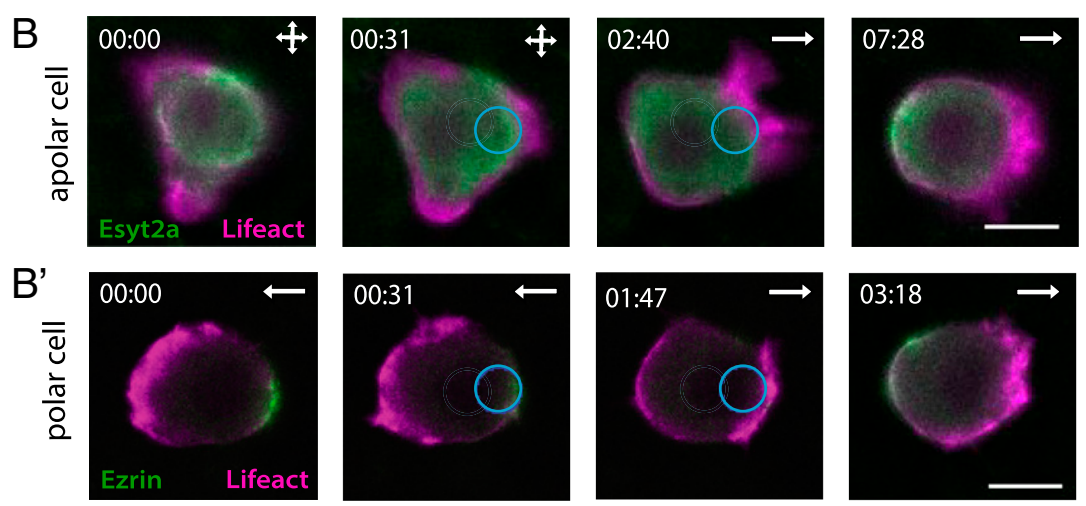

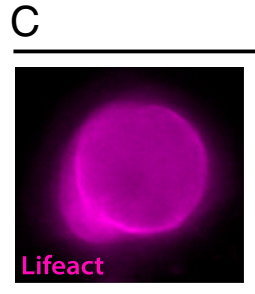

DN-Rac1
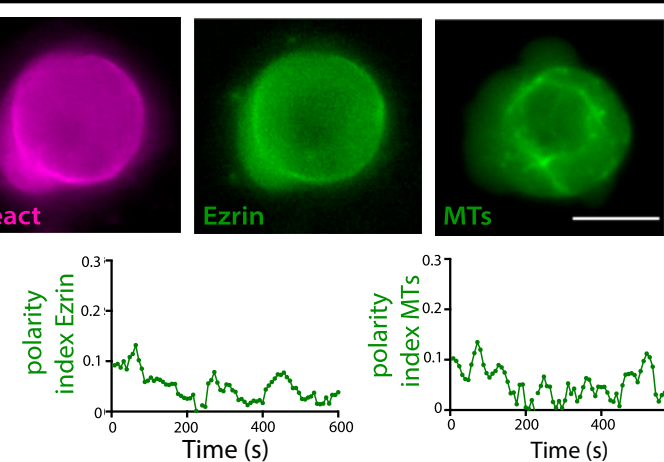

D'
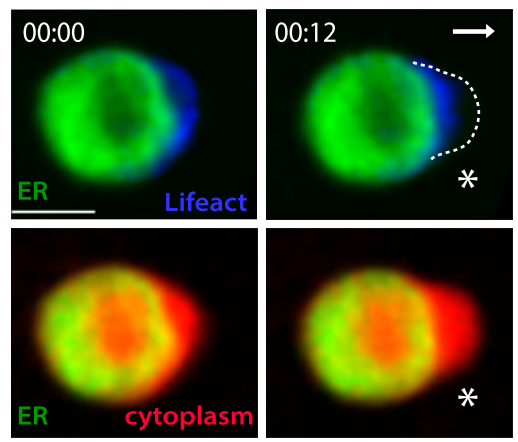
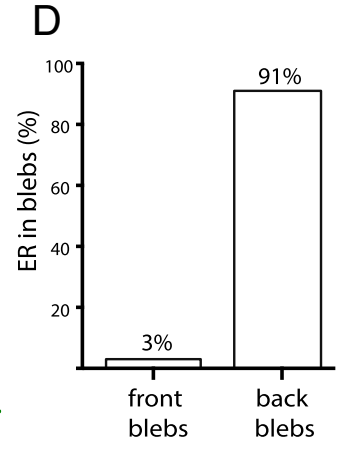

D"
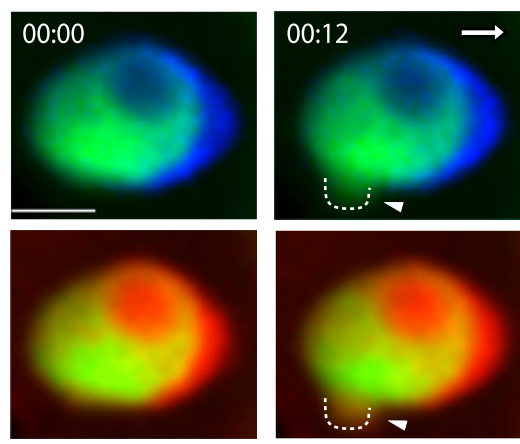

Fig. 3. The role of actin brushes and Rac1 GTPase in the establishment of the self-organizing ligandindependent polarization of PGCs. (A) Polarization of PAK-1 GBD (green), marking locations where Rac1 is active. Dashed line indicates the PAK-1 GBD crescent (Left). Actin brushes (magenta) form at the newly established PAK-1 GBD marked front. $\left(B, B^{\prime}\right)$ Light induced Rac1 activation is sufficient for directing polarization in tumbling PGCs $(B)$ and for reversing polarity in already polarized cells $\left(B^{\prime}\right)$. Lifeact is presented in magenta and Esyt2a and Ezrin fusion proteins are in green. Blue circles indicate the area where the lightactivated Rac1 was activated. (C) Rac1 activity is required for polarity establishment as judged by lack of stable focused accumulation of Lifeact (Left, magenta), Ezrin (Middle, green), and MTs (Right, green, CLIP-170) in cells expressing dominant negative Rac1 (DN-Rac1). Graphs show the polarity index of Ezrin (Left graph) and the MTs (Right graph). (D) Invasion of ER into front and back blebs. $n=140$ front blebs and 29 back blebs in 16 cells. $\left(D^{\prime}, D^{\prime \prime}\right)$ Wild-type PGCs labeled with Lifeact (blue), ER (green), and cytoplasm (red). The blebs are marked by the dashed line. The asterisk indicates the front bleb and the arrowhead points at a bleb formed at the back. (Scale bars, $10 \mu \mathrm{m}$.) Time is in minutes and seconds and white arrows indicate the direction of migration. a physical barrier blocking the entry of ER into the forming bleb (Fig. $3 D^{\prime}$ ). Consistent with this idea is the finding that the ER did enter the blebs at the cell rear (in $91 \%$ of the cases), while only $3 \%$ did so at the leading edge of the cell (Fig. $\left.3 D-D^{\prime \prime}\right)$. The smaller size of the blebs at the back of the cell (SI Appendix, Fig. $\mathrm{S} 1 D$ ) could, in part, be the result of the more viscous ER (43) that resists inflation. This scenario is avoided at the cell front by the presence of actin brushes that prevents organelles from entering the front blebs, thereby controlling their biophysical properties.

Actomyosin-Based Contractility Is Essential for the Establishment of the Cell Rear. To determine the mechanisms that direct the proteins listed above to the cell rear, we investigated their dynamics at the cell periphery. To this end, we made use of the Bioflow software (44). Briefly, Bioflow is a computational tool that allows 
estimating material flow velocities by extracting the motion of fluorescent signals. For this analysis, we followed the dynamics of Esyt2a, benefiting from its more heterogeneous distribution, which is advantageous to tracking. We compared the dynamics of Esyt2a to the movement of a cytoplasmic fluorescent protein that we measured simultaneously. Interestingly, contrary to the cytoplasmic flow to the cell front, Esyt2a flow toward the rear was clearly observed (Fig. $4 A$ and Movie S5). The average flow velocity of the cytoplasmic protein toward the cell front is $0.26 \pm$ $0.10 \mu \mathrm{m}$ per second and the average flow velocity of Esyt2a toward the back of the cell is $-0.16 \pm 0.08 \mu \mathrm{m}$ per second (the minus sign signifies flow in the direction of the rear, five polarized cells, 65 time points). Interestingly, the velocity of both the cytoplasmic flow and that of Esyt2a are increased during bleb expansion (SI Appendix, Fig. S4). This finding can potentially be explained by a front-to-rear actomyosin-dependent retrograde flow $(20,42,45,46)$. To examine whether such a flow could be responsible for the formation of the cell rear in ligandindependent cell polarization, we expressed DN-ROCK, which is known to inhibit actomyosin contraction $(28,47)$. DN-ROCKexpressing PGCs are round and immotile and show no polarization of the back markers (Ezrin, Esyt2a, and MTOC) (Fig. $4 B$ and Movie S6). As judged by the Lifeact signal, the actin brushes, characteristic of the cell front, did form at one side of the cell. When we increased the contractility level, we observed an inverse phenotype; expression of a constitutively active (CA) form of RhoA (RhoAG14V) resulted in a strong retrograde flow and formation of a large stable protrusion at the cell front [Fig. $4 C$, similar to findings in another cell type $(48,49)]$. The strong retrograde flow away from the stable protrusion at the cell front results in very effective transport of Ezrin, Esyt2a, and the MTOC that become homogeneously distributed at the back (Fig. $4 C$ and Movie S7).

Next we sought to elucidate the functional relevance of Ezrin localization to the rear of the cell and specifically tested the possibility that as a membrane-to-cortex linker (29), it prevents blebbing at that region. In agreement with this notion, expression of a hyperactive version of Ezrin (Ezrin 1 to 583) (50) strongly reduced blebbing activity around the cell perimeter (Fig. $4 D$ ). This finding is in line with findings in mesenchymal cells, where the distance between the cell membrane and the actin cortex is reduced at the back, as compared with that at the protruding side of the cell (51).

These results show that an appropriate level of contractility is compatible with cell polarization as reflected in the formation of succession of blebs at the cell front and the definition of the cell rear by Ezrin. The latter stabilizes the cell rear by preventing blebbing at this location.

Orientation of Front-Rear Cell Polarity by Chemokine Signaling. As presented above, polarity of PGCs is established through a selforganizing mechanism that is independent of a guidance cue. Next we sought to determine the mechanism by which a guidance cue orients the front-rear axis with respect to the position of the migration target. To this end, we first introduced a chemokine source next to tumbling apolar PGCs (Fig. 5A) and examined the course of polarity establishment. Interestingly, the order of the polarization events in cells responding to the chemokine was not different from that observed in self-organizing ligand-independent polarization (Fig. $5 B-E$ and $B^{\prime}-D^{\prime}$ and SI Appendix, Fig. S5 $A-C$ and Fig. $2 A-D$ and $A^{\prime}-C^{\prime}$ ). Specifically, we found that the actin brushes formed first, followed by the establishment of the rear as shown by the accumulation of Ezrin, Esyt2a, and MTOC (Fig. $5 B-E$ and Movie S8). Similar to the observation regarding the ligand-independent polarization, the positioning of the MTOC to the rear of the cell represented a late and temporally, highly variable event (ranging between 2.5 and $42 \mathrm{~min}$, Fig. $5 E$ ). It is plausible, therefore, that MTOC localization could in principle be important for persistent migration but not for the initial polarization. These observations are consistent with the idea that the chemokine dictates the positioning of the cell front and the direction of migration simply by biasing an underlying ligandindependent self-organizing polarity cascade. This conclusion was further supported by the findings obtained upon examination of the polarization of dividing PGCs positioned within a chemokine gradient. While the rear markers first accumulated at the cleavage plane as in the ligand independent case, the front formed at the side of the cell facing the higher level of the signal (SI Appendix, Fig. S5 D-F and Movie S9). Under these conditions the formation of the cell front is observed during the last stages of the division before the two daughter cells are fully disconnected. The position of the cell rear was then gradually adjusted according to the position of the new cell front.

To determine the mechanisms by which the activation of Cxcr4b, the Cxcl12a receptor, biases the self-organizing polarity cascade, we monitored the response of manipulated cells to a source of the guidance cue. Considering that Cxcr4b activation leads to an increase in actin polymerization by controlling the Rac1 activation level $(52,53)$, germ cells in which Rac1 activity was inhibited did not polarize at the morphological level, nor did they polarize at the molecular level in response to the chemokine (Fig. $6 A$ and Movie S10). Additionally, when contractility was inhibited by DN-ROCK, cells positioned within a chemokine gradient did not form a cell rear as judged by the apolar distribution of Ezrin and Esyt2a and the positioning of the MTOC (Fig. 6B, SI Appendix, Fig. S6 $A-C^{\prime}$, and Movie S11). Intriguingly however, the majority of cells in which contractility was inhibited (39 out of 49) formed a clear actin-containing front in the direction of the source of the chemoattractant, indicating that they could sense and respond to the chemoattractant (SI Appendix, Fig. S6D). These results show that receptor activation instructs the generation of the cell front, but in order to define a stable front-rear axis with a clear cell back, myosin-dependent retrograde flow is necessary to transport proteins such as Ezrin and Esyt2a. As was the case in the absence of the ligand, increasing the contractility by expressing a constitutively active form of the RhoA protein (RhoAG14V, CA-RhoA) created a stable front protrusion and pushed all the back markers and organelles to the rear (Fig. $6 C$ and Movie S12). In this case, the PGCs did not respond to the chemokine by extending blebs toward the source (Fig. 6D), which reflects the dominance of the strong retrograde flow-induced polarity and depletion of the receptor from the front protrusion (Fig. $6 E$ and $E^{\prime}$ ).

Cellular Self-Polarization Model. Given the findings presented above, we propose the following: The first step in the polarization cascade is the formation of actin brushes, which can be established at any location around the cell perimeter (Fig. $7 \mathrm{~A}$, ii). In the absence of chemokine signaling, this position is determined stochastically. According to our model, the only role of the chemokine is to bias the formation of the actin brushes to a specific site. In this way the actin brushes are more likely to form in the part of the cell facing the higher level of the attractant source. The resulting increase in actomyosin contractility at the site where brushes form could promote breaks in the actin cortex, or push the membrane locally outwards, which would induce weak points of membrane-cortex interaction and favor bleb formation at this aspect of the cell (Fig. $7 A$, iii). In addition, the increased actin retrograde flow at the front, transports membrane linker proteins, such as Ezrin and Esyt2a, away from this region, which increases the likelihood of bleb formation at the forming front. The resulting self-organizing polarization cascade thus defines the rear of the cell by actomyosin-dependent advection of the polarity cues (linker proteins such as Ezrin and Esyt2a) to the opposite side of the cell, where blebbing is therefore inhibited (Fig. $7 A$, iv). The final part of the feedback 

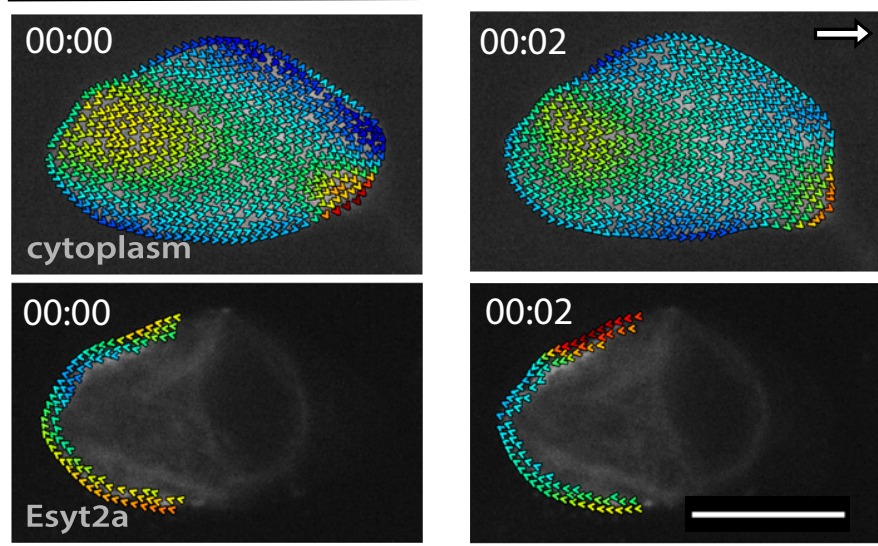

low

velocity

high

B

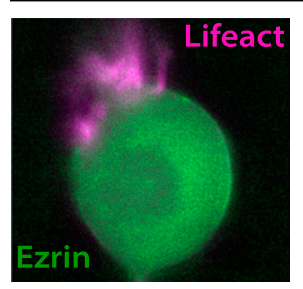

DN-ROCK

C
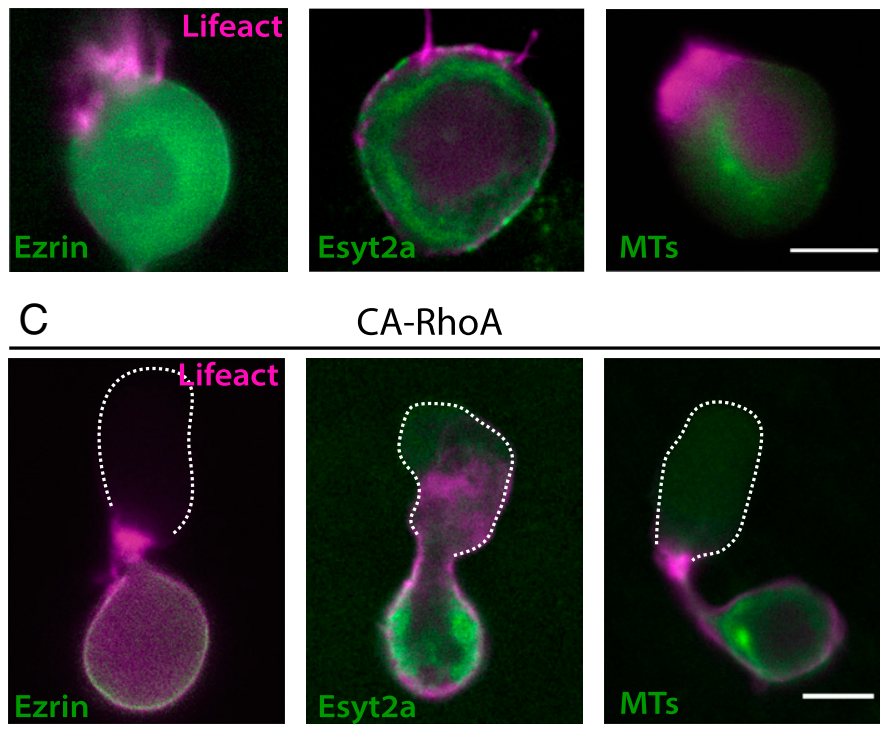

\section{CA-RhoA}

D

Hyperactive Ezrin (1-583)
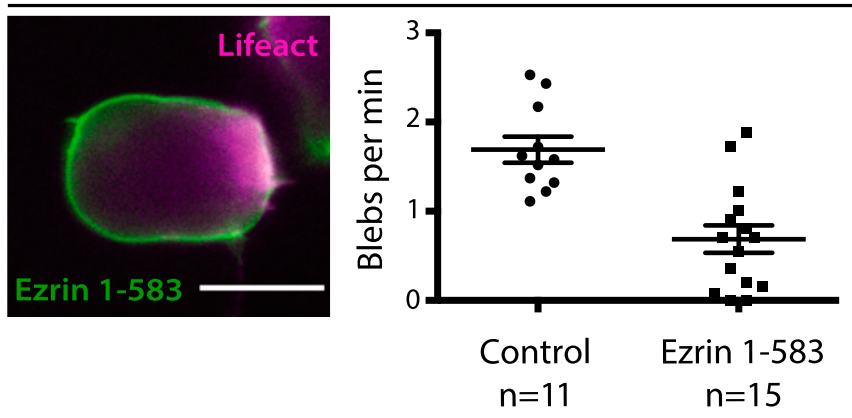

mechanism can be the increased activation of actin polymerization at the sites of blebbing, thereby enhancing the actin flow from the front region. The result of this feedback loop is a stable and robust (persistent) polarization of the cell (Fig. 7B).

To further explain the model for polarization presented above we developed a theoretical model, based on the UCSP model (20). In this model the cell is considered as a one-dimensional
Fig. 4. The role of contractility and of Ezrin function in ligandindependent self-organized polarization of PGCs. $(A)$ Intracellular flow fields of cytoplasm and Esyt2a during PGC migration in wild-type embryos, measured using Bioflow software. White arrow indicates the direction of migration. (B) ROCK activity is required for the establishment of the rear. Lifeact is in magenta and Ezrin (Left), Esyt2a (Middle), and MTs (Clip170H, Right) are in green in cells expressing a DN-ROCK. (C) Enhanced contractility induced by expression of a constitutively active RhoA (CA-RhoA) results in hyperpolarization of the cell. Lifeact is in magenta and Ezrin (Left), Esyt2a, (Middle), and MTs (Clip170H, Right) are in green. Dashed line marks the contours of the cell front. $(D)$ Hyperactive version of Ezrin (amino acids 1 to 583) is distributed over a larger portion of the cell perimeter (Left, Lifeact in magenta and Ezrin in green) and inhibits blebbing activity (graph on the Right, Ezrin (1 to 583) $n$ is the number of cells, twotailed $t$ test with Welch's correction, $P=<0.0001$ ). (Scale bars, $10 \mu \mathrm{m}$.)

object of fixed length, with two opposing ends where treadmilling actin originates. The net actin treadmilling in the cell is due to the difference between the flow that originates at either end and causes the advection of various proteins that bind actin (SI Appendix, Fig. S7A). When these proteins affect the process that initiates actin treadmilling flow at the cell ends, positive feedback loops can drive the cell to spontaneously self-polarize. Myosin II 
A

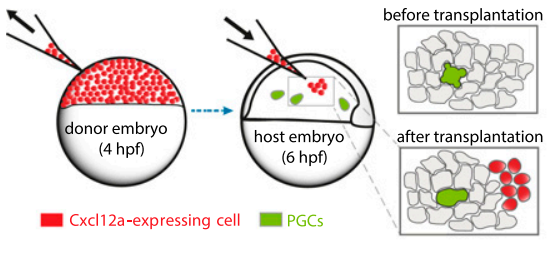

E Chemokine-directed cell polarization after tumbling

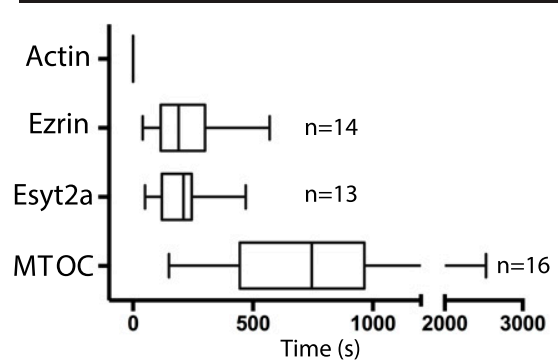

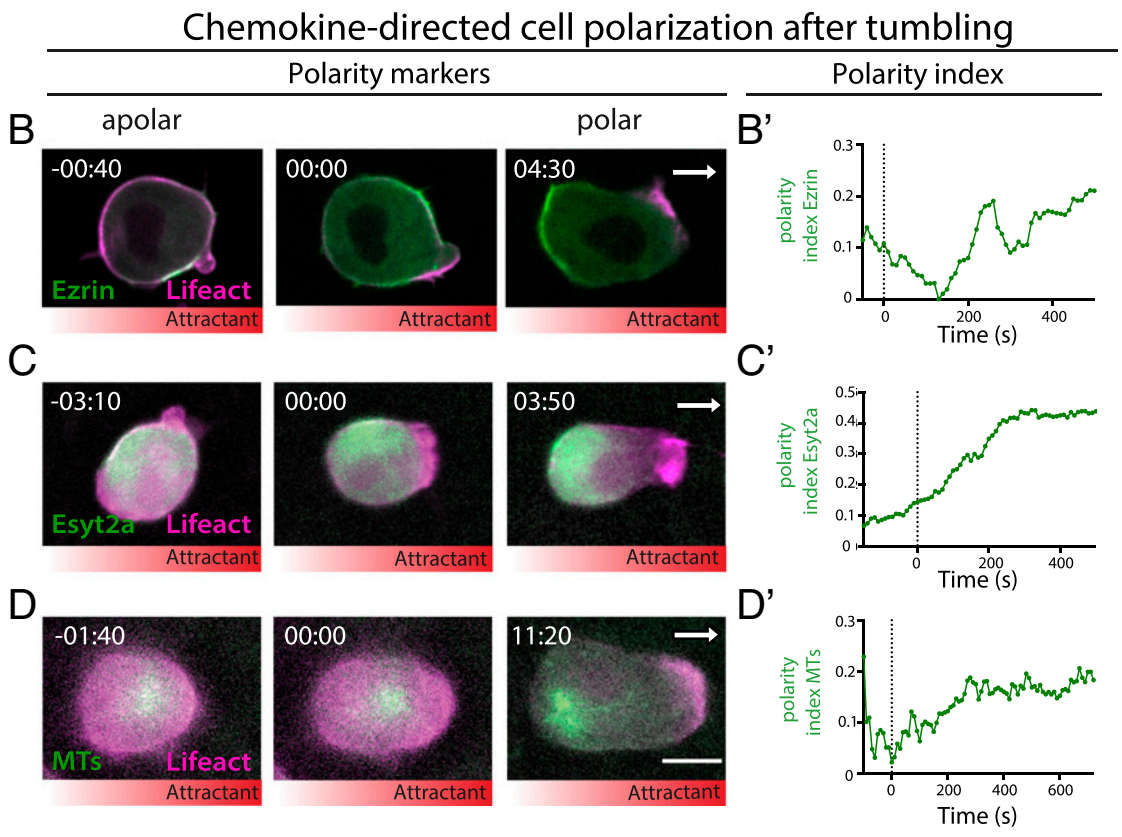

Fig. 5. Chemokine-directed polarity establishment. (A) A scheme presenting the generation of local chemokine source within the live embryo. (B-D) Apolar cells (Left), the emergence of the polarized front (time 00:00) (Middle), and the subsequent polarized localization of the rear markers (Right). Lifeact is labeled with magenta and rear markers $(B)$ Ezrin, $(C)$ Esyt2a, and $(D)$ MTs (Clip170H) are in green. White arrows indicate the direction of migration toward the chemokine source. Time is in minutes and seconds. (Scale bar, $10 \mu \mathrm{m}$.) $\left(B^{\prime}-D^{\prime}\right)$ Time course of polarity index establishment during cell polarization; time is in seconds. For $B^{\prime}$ and $C^{\prime}$ the signal was measured only at the level of the membrane. In $B-D$ the distribution of the chemokine $(\mathrm{Cxcl} 12 \mathrm{a})$ is illustrated by the red gradient below the panels. $(E)$ Timing of the chemokine induced polarization steps relative to the time of actin localization at the cell front (time 0) (median, 5 and 95 percentiles are presented, $n=$ number of cells, time in seconds). is a protein that is advected by the actin flow to the cell rear, while its contractile force further enhances the flow in the direction of the rear end. Here we present another mechanism for the self-polarization, namely the advection and enrichment of proteins such as Ezrin and Esyt2a, which inhibit the blebbing activity at the cell edge. By advecting these proteins away from the dominant leading edge, this aspect of the cell becomes more bleb prone, while blebbing at the opposing part of the cell is inhibited. If blebbing supports the maintenance of enhanced actin polymerization (SI Appendix, Fig. S2D), the linker proteins effectively suppress maintenance of actin polymerization at the rear end of the cell by inhibiting blebbing. This positive feedback can be described by the same set of equations previously proposed in refs. 20, 21, and naturally explains many of the observations reported above.

According to this model, when the positive feedback reaches a critical threshold, the uniform system becomes unstable, and small spontaneous fluctuations in actin polymerization are enhanced until a polarized state is established. In the polarized state the actin flow is asymmetric, strong at the front and weak at the back, together with an exponential distribution of the inhibitory proteins that accumulate at the back (SI Appendix, Fig. $\mathrm{S} 7 A$ ). The model predicts a phase diagram (SI Appendix, Fig. $\mathrm{S} 7 B$ ), relating the polarization state of the cell to the total level of inhibitory proteins and strength of the actin retrograde flow. In particular, there is a range of parameters where the model predicts an "intermittent" phase, where cells exhibit run-andtumble migration due to stochastically losing (and regaining) polarization. This behavior arises when the model solutions are bistable, with the possibility for the development of polarized and unpolarized states as observed in PGCs.

The model we propose predicts the dependency of cell polarization on several key components. First, below a critical value of the treadmilling actin flow, there is no self-polarization, as the positive feedback mechanism is too weak. Indeed, when DNROCK is expressed, the contractile forces are weaker, and so is the treadmilling flow, such that the cell remains unpolarized (Figs. $4 B$ and $6 B$ ). Second, high levels of Ezrin activity compromise cell polarity (Fig. $4 D$ ). In this theoretical model, it is when the total amount of inhibitor (of Ezrin in this case) is too high, cell polarity is globally inhibited. These transitions from the motile (polarized) to the nonmotile (unpolarized) cell conditions are presented qualitatively in SI Appendix, Fig. S7B. The model also explains the formation of a polarized cell when two competing fronts exit initially (21) (Fig. $3 B$ and SI Appendix, Fig. S7A).

Finally, our model predicts a relation between the value of the polarity $(\mathrm{P})$ of the protein that is advected by the actin flow and its effective diffusion coefficient (D) such that $\mathrm{P}$ is proportional to 1/D (SI Appendix, Fig. S7C). The time scale for reaching the steady-state distribution of the protein following the beginning of the advection is also expected to depend inversely on the effective diffusion coefficient. Therefore, we expect that proteins that diffuse faster will reach their steady-state distribution more quickly and have lower polarity index, compared to proteins that have lower effective diffusion coefficients. This relation indeed appears qualitatively in Fig. $2 A^{\prime}$ and $B^{\prime}$ and $5 B^{\prime}$ and $C^{\prime}$ for Ezrin 

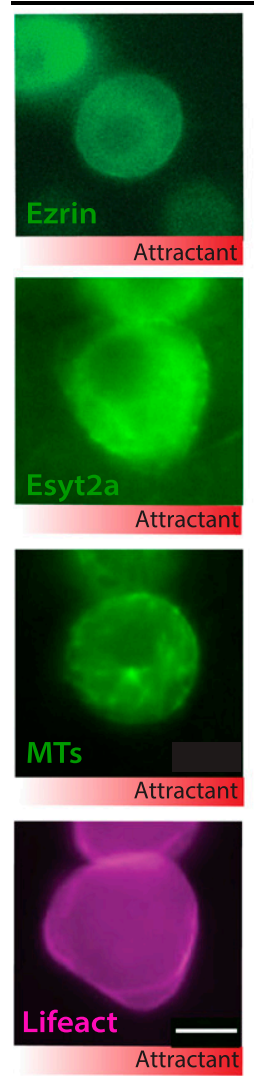

DN-Rac1

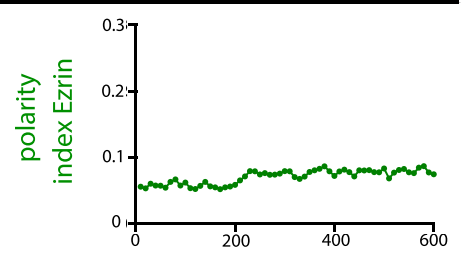

Time (s)
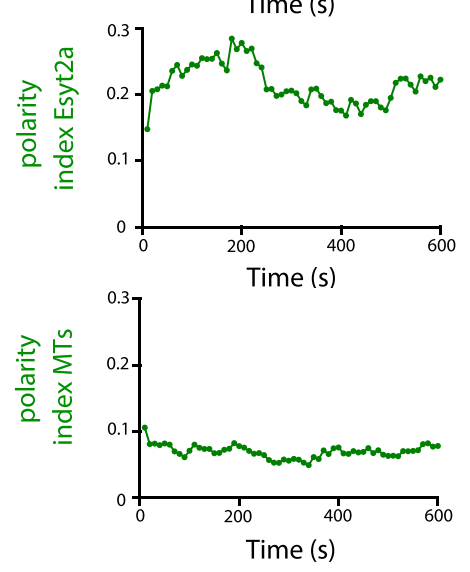

B

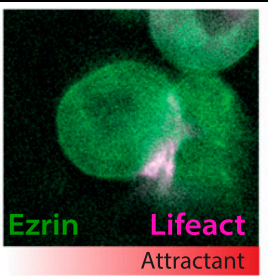

C
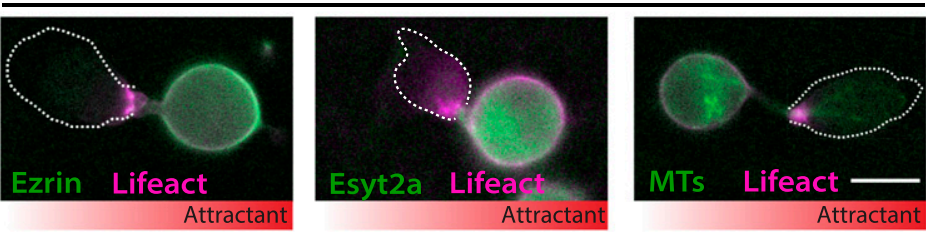

Direction of the front protrusion relative to the position of the chemokine source
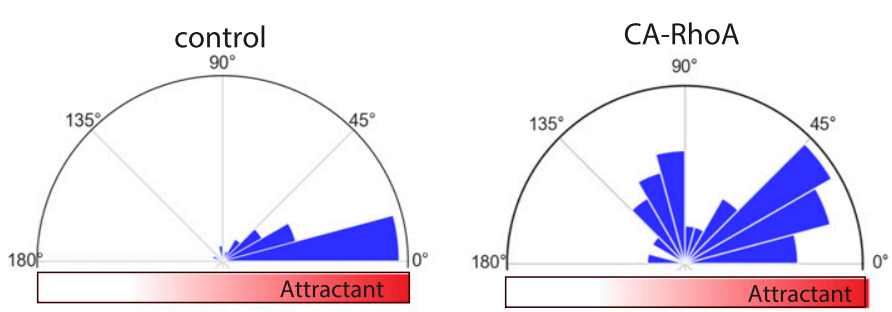
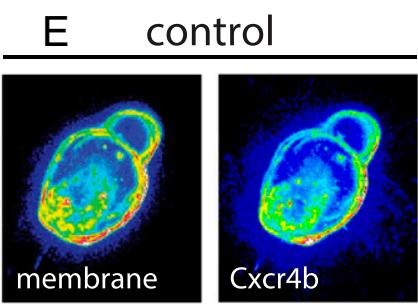

\section{E' CA-RhoA}

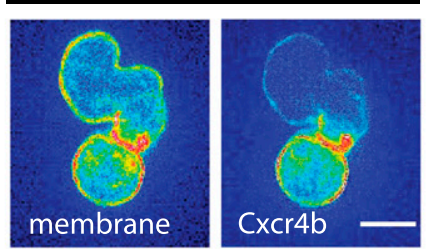

Fig. 6. Mechanism of chemokine-induced polarization. (A) Rac1 activity is required for ligand-induced polarity establishment. Localization of Ezrin (green, Upper), Esyt2a (green, second panel), MTs (green, third panel), and Lifeact (magenta, Lower) in DN-Rac1-expressing PGCs in the presence of a chemokine gradient. Graphs show the polarity index of Ezrin (Upper), Esyt2a (Middle), and the MTs (Lower). (B) Localization of Ezrin (green, Left), Esyt2a (green, Middle), and MTs (green, Right) in DN-ROCK-expressing PGCs in the presence of a chemokine gradient. Lifeact is marked in magenta. Inhibition of contractility by DNROCK affects the definition of the cell rear in these cells. (C) Enhancement of contractility by expression of a constitutively active RhoA (CA-RhoA) in the presence of the chemokine gradient with Lifeact (magenta) and Ezrin (Left), Esyt2a (Middle), and MTs (Right) in green. (D) The angle of protrusions relative to the position of the attractant source in wild-type cells (Left, $n=72)$, and in PGCs with increased contractility (Right, $n=50)$. In the rose plot, $0^{\circ}$ indicates the source of the chemokine. ( $E$ and $\left.E^{\prime}\right)$ Cxcr4b expression in control $(E)$ and CA-RhoA-expressing cells $\left(E^{\prime}\right)$. The plasma membrane is labeled with a farnesylation signal and Cxcr4b is an in-frame fusion with GFP (z-projection). In $A-D$ the distribution of the chemokine (Cxcl12a) is illustrated by the gradient in the red rectangle. (Scale bars, $10 \mu \mathrm{m}$.)

and Esyt2a, where the proteins that take a longer time to reach steady-state reach higher values of the polarity $P$. The effective diffusion coefficient of Esyt2a may be small due to transient binding of the protein to the ER that pervades the cytoplasm. Together, the theoretical model presented above explains the observed experimental results and can provide predictions regarding the robustness and the behavior of the cell under different conditions.

\section{Discussion}

Studies describing the polarization of migrating cells primarily reveal the role of biochemical signaling pathways involving small GTPases, GEFs and PIP3 signaling that control actin polymerization and define the cell front $(52,54,55)$. The cell front was suggested to direct the formation of the rear at the other aspect of the cell, for example by activating contractility away from it or by inducing flow of actin polymerization inhibitors away toward the forming rear $(8,56)$. Here, we investigated the establishment of a front-rear axis in bleb-driven migration in vivo in the context of the developing embryo. At the exit from tumbling, filamentous actin is the first structure to appear in the polarization cascade whether the attractant Cxcl12 is present or not, similar to findings in Dictyostelium cells that rapidly respond to graded distribution of the attractant by actin polymerization (57). While the role of actin in pseudopod formation is to push the membrane forward, based on studies in Dictyostelium, its role in bleb formation is to introduce negative curvature to the membrane that favors bleb formation (34). Our analysis of migrating PGCs suggests that actin brushes have additional functions. They serve as a scaffold for the accumulation of proteins such as MLCK 


\section{A}

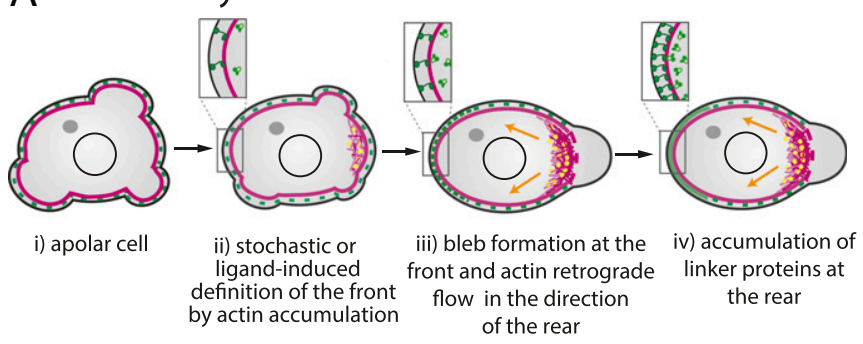

B Polarity maintenance

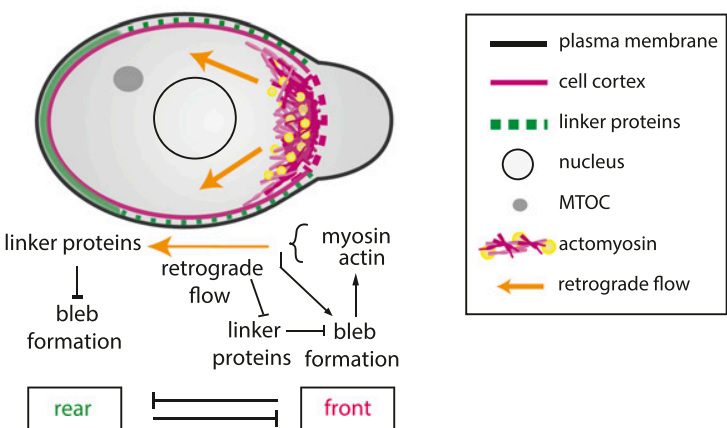

Fig. 7. A model for polarity establishment and maintenance in migrating PGCs. (A) The exit from the apolar state ( $i$ ) can either be in a random direction, or biased by the polar distribution of the guidance cue. As judged by the site of actin polymerization, the cell front is defined first (ii). Actin accumulation at the front focuses the protrusions to the same location and actomyosindependent retrograde flow leads to progressive increase in the amount of the linker proteins (Ezrin and Esyt2a, green) and the Septin9a protein at the rear (iii). In the fully polarized cell, high levels of linker proteins are found at the cell rear (iv). (B) Polarity is maintained by the enhanced contractility at the cell front that promotes bleb formation at the front and flow of bleb-inhibiting linker proteins to the rear, where they inhibit bleb formation.

(SI Appendix, Fig. S2 $A$ and $B$ ), whose activation could introduce breaks in the cortex, thereby facilitating inflation of the bleb that occurs by unfolding of membrane invaginations (35). Second, the location of the brushes marks the position from which retrograde flow toward the other side of the cell is initiated to establish the cell back. Last, the brushes serve as a barrier for organelles and structures such as the ER, which could affect the biophysical properties of protrusions and their size (Fig. 3D and SI Appendix, Fig. S1 $C$ and $D$ ). An interesting possibility concerning the maintenance of the cell front is that the formation of blebs positively influences actin polymerization. Such a positive feedback scenario is consistent with our finding that the stability of actin brushes is reduced in cells in which blebbing is inhibited (SI Appendix, Fig. S2D). According to this hypothesis, unfolding of membrane invaginations during bleb formation (35) could release factors that in turn promote actin polymerization (58).

Our work underscores the importance of the transport of molecules that change biophysical parameters in the course of polarity establishment, in particular the advection of linker molecules that inhibit blebbing at the cell rear. Ezrin inhibits blebbing by directly increasing the attachment of the plasma membrane to the cell cortex (Fig. 4D). Extended synaptotagminlike $2 \mathrm{a}$ on the other hand, could inhibit blebbing indirectly by linking the plasma membrane to the membrane of the endoplasmic reticulum, which would limit bleb expansion at the cell rear (Fig. 3D and SI Appendix, Fig. S1D). Additionally, these linker proteins could also play a role in polarization of the cells by increasing membrane tension at the rear (e.g., Ezrin) (59), a biophysical feature that was shown to play a role in maintenance of the front of migrating neutrophils $(8,60)$. Our analysis suggests that the transport of these molecules to the cell rear is driven by retrograde flow generated by actomyosin contractility. The advection of these linker molecules could depend on direct interaction with actin that flows toward the cells back (e.g., for Ezrin) (30), or other yet to be identified mechanisms (e.g., for Esyt2a and Septins).

Our data reveal that during the establishment of cell polarity at the exit from the apolar tumbling phases the front is defined first, with the rear forming at the other side of the cell a few minutes later, in a process that depends on actomyosin contractility. Consistent with the reciprocal inhibition between the front and the rear of the cell, when the rear forms first, as is the case following cell division, the front is defined at the opposite side of the cell in a process of self-organizing polarity establishment. Interestingly, we observed that the time required for PGCs to polarize is remarkably similar whether the cells polarize spontaneously or in response to a chemokine gradient. The results we present are therefore consistent with the idea that the role of the graded distribution of the chemokine is merely to bias the initial definition of the actin polymerization site that is followed by the development of the rear.

Retrograde flow positions linker molecules at one aspect of the cell where they inhibit blebbing, to establish the rear of the cell. At the same time, the low abundance of these linkers at the opposite side of the cell defines a region that is more permissive to blebbing, the cell front. These events lead to the development of self-enhancing polarization that can be initiated by small fluctuations in actin polymerization leading to nondirectional migration, or by biased actin polymerization in response to chemotactic cues resulting in directed migration. The ensuing robust polarization of the cell (Fig. $7 B$ ) raises a problem concerning the introduction of corrections to the migration path. In the case of germ cells, such changes in migration direction are made possible following tumbling phases, which are periodic events of loss of cell polarity (27). We showed that such a run-and-tumble behavior can arise naturally in our theoretical model (SI Appendix, Fig. S7B) (21), in a regime when both polarized and unpolarized states can develop.

During their normal and ligand-independent migration, PGCs can reach almost any part of the embryo, which consists of a wide variety of cell and tissue types that exhibit different biophysical properties $(26,61,62)$. This scenario is similar to that observed for other cell types that form blebs and migrate through complex environments within the body (e.g., cancer cells and neutrophils) $(13,14)$. It would be interesting to examine to what degree the model we propose here and the role of specific molecules are relevant for other cell types that use blebs to propel their migration.

\section{Materials and Methods}

Zebrafish Strains and Maintenance. Zebrafish (Danio rerio) embryos of the $A B$ and $A B / T L$ backgrounds and embryos from other transgenic fish lines were used as wild-type embryos. Zebrafish were maintained according to the recommendations in 2007/526/EC and Article 33 of European Union Directive 2010/63 (63). For a list of the fish lines used in this work, see SI Appendix, Table S1.

Generation of Transgenic Fish Lines. Transgenic fish lines expressing Ezrin, Esyt2a, Clip170H, or Septin9a in PGCs, alone or in combination with Lifeact or a farnesylated fluorescent protein, were generated (SI Appendix, Table S2). Plasmids containing the askopos (kop) promoter (64), the protein of interest fused to a fluorophore, the nanos3 3' UTR and a dominant marker were generated using Gateway Technology (Invitrogen). The plasmid was injected with RNA for Tol2.3 transposase into the cell of one-cell-stage embryos, and adult fish were screened for the dominant marker transmission in their progeny. Fish carrying the kop:lifeact.mCherry.nos3'UTR-cry:DsRed were crossed to fish carrying the kop:YPET.Ezrin.nos3'UTR-cmlc:GFP and fish carrying kop:mTFP1.Septin9a.nos3'UTR$c m / c: E G F P$ to generate the fish lines carrying both transgenes.

RNA and Morpholino Antisense Oligonucleotides Microinjection. One-cell-stage zebrafish embryos were microinjected into the yolk with $1 \mathrm{~nL}$ of either sense 
mRNA and/or a translation blocking morpholino antisense oligonucleotide (MO GeneTools). mRNA was synthetized using the mMessageMachine kit (Ambion) A list of constructs used in this study and the mRNA concentration are provided in SI Appendix, Tables S3 and S4. To express proteins in germ cells, the corresponding sequence was cloned upstream of the nanos3 3' UTR (65). For global protein expression the sequence was cloned upstream of a Xenopus globin 3 UTR. Embryos were kept in $0.3 \times$ Danieau's solution and incubated at $25^{\circ}$.

Image Acquisition. Fluorescence microscopy was performed using a Carl Zeiss Axio Imager Z1 equipped with a Retiga R6 camera or a Carl Zeiss Axio Imager Z1 equipped with a Spot Pursuit 23.0 1.4 MP monochrome camera. Spinning disk microscopy was performed using a Carl Zeis Axio Imager Z1 coupled to a Yokogawa CSUX1FW-06P-01 spinning disk unit and a Hamamatsu OrcaFlash4.0LT C11440 camera. Time-lapse movies were acquired using VisiView software. The confocal microscopy images were generated using a Car Zeiss LSM 710 microscope and Zen software. PGC imaging was performed between $60 \%$ and $90 \%$ epiboly stages. Frames were captured at 8 -s intervals for experiments performed without the chemokine and 10-s intervals were used for the experiments with the chemokine, with the exception of experiments in Figs. $3 B$ and $D$ and $4 A$ in which frames were captured at $7.75,2$ and $0.5 \mathrm{~s}$, respectively. Embryos were kept at $28^{\circ}$ during image acquisition.

Quantification of Bleb Location and Size. The location of blebs was determined based on the position of the bleb center relative to the cell center. The center of mass (COM) of the cell was determined using FIJI software (NIH). The center of cell front was defined based on the COM of the actin brushes (labeled by Lifeact-GFP). The bleb perimeter was drawn based on the membrane signal (labeled with farnesylated mCherry) and the COM was calculated in FIJI. The angle between the line connecting the COM of the bleb to the COM of the cell and the line connecting the COM of the cell front to the COM of the cell was measured in FIJI. Front blebs were defined as having angles smaller than $45^{\circ}$ with respect to the COM of the actin brushes and rear blebs as showing angles larger than $135^{\circ}$. All other angles were referred to as side blebs.

Imaging of Self-Organizing Polarity in PGCs. PGCs were imaged between $60 \%$ and $90 \%$ epiboly using epifluorescence or spinning disk microscopy. One-cellstage embryos were injected with RNA encoding for the specific polarity markers and/or MO oligonucleotides directed against Cxcl12a (21) (GeneTools) (SI Appendix, Table S5). Images were processed using FIJI. For quantification of the polarization times, time 0 was considered to be when the actin brushes were first detected. The cascade of generation of blebs and polarization of other proteins and structures (Ezrin, Esyt2a, Septin9a, and MTOC) was followed.

Imaging of Chemokine-Dependent Polarization of PGCs. These experiments were performed by transplantation of somatic cells expressing $\mathrm{CxCl} 12 \mathrm{a}$ into host embryos depleted of the chemokine using a morpholino against $c x c / 12 a$. PGCs of host embryos were labeled for the different polarity markers. Cells from donor embryos (between $30 \%$ epiboly and shield stages) were transplanted into host embryos (between shield and $75 \%$ epiboly stages) on a Carl Zeiss Axio Imager Z1 microscope with a $5 \times$ objective and immediately followed by the acquisition of time-lapse movies using either a $40 \times$ or $63 \times$ objective. Image processing and quantification were performed as described above for the ligand-independent self-organizing polarity.

Protein Polarization and Polarity Difference Angle Calculation. To determine the angular polarity index, we generated an intensity-based Matlab (Mathworks) program that follows the approach described in a recent study (35) (for details see SI Appendix, Extended Methods, Protein Polarization and Polarity Difference Quantification).

Photoactivatable Rac1-Based Experiments. MO directed against cxc/12a, EzrinYPet.nos3'UTR RNA, or Ypet.esyt2al.nos RNA and either the photoactivatable version of Rac1 fused to the 3' UTR of nanos3 or control RNA were injected in one-cell-stage kop:lifeact.mCherry.nos3'UTR-cry:DsRed embryos. The $63 \times$ movies were acquired using a Carl Zeiss LSM 710 confocal

1. J. P. Campanale, T. Y. Sun, D. J. Montell, Development and dynamics of cell polarity at a glance. J. Cell Sci. 130, 1201-1207 (2017)

2. L. E. Hind, W. J. B. Vincent, A. Huttenlocher, Leading from the back: The role of the uropod in neutrophil polarization and migration. Dev. Cell 38, 161-169 (2016).

3. E. Infante, A. J. Ridley, Roles of Rho GTPases in leucocyte and leukaemia cell transendothelial migration. Philos. Trans. R. Soc. Lond. B Biol. Sci. 368, 20130013 (2013).

4. M. Abercrombie, J. E. M. Heaysman, S. M. Pegrum, The locomotion of fibroblasts in culture. I. Movements of the leading edge. Exp. Cell Res. 59, 393-398 (1970). microscope and the photoactivation was performed using the bleaching module in the Zen software. Photoactivation was initiated after five frames with a 456-nm laser using a circular region of interest (ROI) with a diameter of $5 \mu \mathrm{m}$ (120 to 240 iterations, $100 \%$ laser power) and was repeated every four frames. The ROI was located at the edge of the cell opposite to existing actin brushes or at a random location in apolar cells.

Overexpression of N-BAR Domain, DN-Rac1, DN-ROCK, CA-RhoA, and Hyperactive Ezrin Proteins. Embryos of transgenic fish expressing polarity markers or $A B$ fish were injected with RNA encoding for polarity markers, the dominant-negative or constitutively active proteins fused to the nanos3'UTR and MO directed against $c x c / 12 a$. PGCs were imaged between shield and $90 \%$ epiboly stages, either in the absence of Cxcl12a or in the presence of transplanted cells producing the chemokine. For overexpression of N-BAR domain, imaging was conducted at 17 to 19 hours post fertilization (hpf). Only non-blebbing cells (16 of 35 cells) were selected for the analysis.

Quantification of Extended Synaptotagmin-Like 2a Flow Field. To analyze the flow of extended synaptotagmin 2a (Esyt2a), we expressed the YPet-Esyt2a and mTomato, as a cytoplasmic control, in migrating PGCs. Imaging was performed using spinning disk microscopy and frames were captured every $500 \mathrm{~ms}$. The two channels corresponding to Esyt2a and the cytoplasm were analyzed using an adaptation of BioFlow (44) in the Icy platform (66) to derive velocity fields within the segmented cells from consecutive pairs of images over a time period (for details see SI Appendix, Extended Methods, Flow Field Quantification).

Direction of the Front in PGCs Expressing CA-RhoA Relative to the Chemokine Gradient. Cell transplantation was used to generate a local source of the chemokine Cxcl12a. Donor embryos were injected with RNA encoding for $\mathrm{Cxcl} 12 \mathrm{a}$. The host embryos were injected MO directed against $\mathrm{cxc} / 12 \mathrm{a}$ and either control RNA or RNA encoding for CA-RhoA targeted to the PGCs by the nanos3 3'UTR. Embryos were imaged starting from 30 min after transplantation. The direction of the front relative to the chemokine source was determined by measuring the angle between the line connecting the position of the chemokine source to the center of the cell and the line connecting the center of the cell to the cell front. An angle of $0^{\circ}$ indicated perfect alignment of the front toward the chemokine source. The angles were plotted into a rose plot generated in Python.

Deconvolution. The time lapse movies from Figs. $1 F$ and $G$, and $2 A$ and $B$, and $3 A$ and $S I$ Appendix, Fig. S3D were deconvolved using the software Huygens Professional.

Statistics and Reproducibility. Statistical analysis was conducted using GraphPad Prism 6.0d software. The statistical test used, the $n$ and the $P$ values are indicated in the figure legends. All experiments were repeated at least three times, except for the analysis of the persistence of actin brushes presented in SI Appendix, Fig. S2D, which was performed twice.

Data Availability. All study data are included in the article and/or supporting information.

ACKNOWLEDGMENTS. This work was supported by the European Research Council (ERC, CellMig, no. 268806 to E.R. and PolarizeMe, no. 771201 to T.B.), the Deutsche Forschungsgemeinschaft (DFG, RA863/11-1, SFB 1348, and CRU326), and the Cells in Motion Cluster of Excellence (EXC 1003-CIM). N.S.G. is the incumbent of the Lee and William Abramowitz Professorial Chair of Biophysics and this research was supported by the Israel Science Foundation (Grant 1459/17). A.B.-P. is a member of the Pasteur-Paris University (PPU) International PhD Program, funded by the European Union's Horizon 2020 Research and Innovation Programme under the Marie Skłodowska-Curie grant agreement no. 665807, and by the Institut Carnot Pasteur Microbes \& Santé (ANR 16 CARN 0023-01). We thank Klaus Hahn for the photoactivatable Rac construct. We thank Nina Knübel for the initial design of the model and Celeste Brennecka for critical reading of the manuscript. We thank Bart Vos for the Python script used for the rose plot.

5. M. Abercrombie, J. E. M. Heaysman, S. M. Pegrum, The locomotion of fibroblasts in culture. 3. Movements of particles on the dorsal surface of the leading lamella. Exp. Cell Res. 62, 389-398 (1970)

6. J. M. Nichols, D. Veltman, R. R. Kay, Chemotaxis of a model organism: Progress with Dictyostelium. Curr. Opin. Cell Biol. 36, 7-12 (2015).

7. A. Shellard, R. Mayor, Integrating chemical and mechanical signals in neural crest cell migration. Curr. Opin. Genet. Dev. 57, 16-24 (2019).

8. A. R. Houk et al., Membrane tension maintains cell polarity by confining signals to the leading edge during neutrophil migration. Cell 148, 175-188 (2012). 
9. N. Andrew, R. H. Insall, Chemotaxis in shallow gradients is mediated independently of Ptdlns 3-kinase by biased choices between random protrusions. Nat. Cell Biol. 9, 193-200 (2007).

10. J. Mueller et al., Load adaptation of lamellipodial actin networks. Cell 171, 188-200.e16 (2017).

11. A. Diz-Muñoz, D. A. Fletcher, O. D. Weiner, Use the force: Membrane tension as an organizer of cell shape and motility. Trends Cell Biol. 23, 47-53 (2013).

12. O. T. Fackler, R. Grosse, Cell motility through plasma membrane blebbing. J. Cell Biol. 181, 879-884 (2008).

13. T. Lämmermann, M. Sixt, Mechanical modes of 'amoeboid' cell migration. Curr. Opin. Cell Biol. 21, 636-644 (2009).

14. A. Laser-Azogui, T. Diamant-Levi, S. Israeli, Y. Roytman, I. Tsarfaty, Met-induced membrane blebbing leads to amoeboid cell motility and invasion. Oncogene 33 1788-1798 (2014)

15. E. K. Paluch, E. Raz, The role and regulation of blebs in cell migration. Curr. Opin. Cell Biol. 25, 582-590 (2013).

16. G. T. Charras, C.-K. Hu, M. Coughlin, T. J. Mitchison, Reassembly of contractile actin cortex in cell blebs. J. Cell Biol. 175, 477-490 (2006).

17. M. Goudarzi, A. Boquet-Pujadas, J.-C. Olivo-Marin, E. Raz, Fluid dynamics during bleb formation in migrating cells in vivo. PLoS One 14, e0212699 (2019).

18. M. Goudarzi et al., Identification and regulation of a molecular module for blebbased cell motility. Dev. Cell 23, 210-218 (2012).

19. I. Lavi et al., Cellular blebs and membrane invaginations are coupled through membrane tension buffering. Biophys. J. 117, 1485-1495 (2019).

20. P. Maiuri et al., Actin flows mediate a universal coupling between cell speed and cell persistence. Cell 161, 374-386 (2015).

21. J. E. Ron, P. Monzo, N. C. Gauthier, R. Voituriez, N. S. Gov, One-dimensional cell motility patterns. Phys. Rev. Research 2, 033237 (2020).

22. T. Mitchison, M. Kirschner, Cytoskeletal dynamics and nerve growth. Neuron 1 761-772 (1988)

23. V. Swaminathan, C. M. Waterman, The molecular clutch model for mechano transduction evolves. Nat. Cell Biol. 18, 459-461 (2016).

24. T. W. Anderson, A. N. Vaughan, L. P. Cramer, Retrograde flow and myosin II activity within the leading cell edge deliver F-actin to the lamella to seed the formation of graded polarity actomyosin II filament bundles in migrating fibroblasts. Mol. Biol. Cell 19, 5006-5018 (2008).

25. A. Paksa, E. Raz, Zebrafish germ cells: Motility and guided migration. Curr. Opin. Cell Biol. 36, 80-85 (2015).

26. M. Doitsidou et al., Guidance of primordial germ cell migration by the chemokine SDF-1. Cell 111, 647-659 (2002).

27. M. Reichman-Fried, S. Minina, E. Raz, Autonomous modes of behavior in primordia germ cell migration. Dev. Cell 6, 589-596 (2004)

28. H. Blaser et al., Migration of zebrafish primordial germ cells: A role for myosin contraction and cytoplasmic flow. Dev. Cell 11, 613-627 (2006).

29. S. Martinelli et al., Ezrin/Radixin/Moesin proteins and flotillins cooperate to promote uropod formation in T cells. Front. Immunol. 4, 84 (2013).

30. M. Arpin, D. Chirivino, A. Naba, I. Zwaenepoel, Emerging role for ERM proteins in cell adhesion and migration. Cell Adhes. Migr. 5, 199-206 (2011).

31. R. Fernández-Busnadiego, Y. Saheki, P. De Camilli, Three-dimensional architecture of extended synaptotagmin-mediated endoplasmic reticulum-plasma membrane contact sites. Proc. Natl. Acad. Sci. U.S.A. 112, E2004-E2013 (2015).

32. F. Giordano et al., $\mathrm{PI}(4,5) \mathrm{P}(2)$-dependent and $\mathrm{Ca}(2+)$-regulated ER-PM interactions mediated by the extended synaptotagmins. Cell 153, 1494-1509 (2013).

33. M. Lam, F. Calvo, Regulation of mechanotransduction: Emerging roles for septins Cytoskeleton (Hoboken) 76, 115-122 (2019).

34. S. Collier, P. Paschke, R. R. Kay, T. Bretschneider, Image based modeling of bleb site selection. Sci. Rep. 7, 6692 (2017)

35. M. Goudarzi et al., Bleb expansion in migrating cells depends on supply of membrane from cell surface invaginations. Dev. Cell 43, 577-587.e5 (2017).

36. A. J. Ridley et al., Cell migration: Integrating signals from front to back. Science 302, 1704-1709 (2003)

37. B. R. Graziano et al., Cell confinement reveals a branched-actin independent circuit for neutrophil polarity. PLOS Biol. 17, e3000457 (2019).

38. A. M. Hebert, B. DuBoff, J. B. Casaletto, A. B. Gladden, A. I. McClatchey, Merlin/ERM proteins establish cortical asymmetry and centrosome position. Genes Dev. 26, 2709-2723 (2012)
39. U. G. Knaus, G. M. Bokoch, The p21Rac/Cdc42-activated kinases (PAKs). Int J. Biochem. Cell Biol. 30, 857-862 (1998).

40. Y. I. Wu et al., A genetically encoded photoactivatable Rac controls the motility of living cells. Nature 461, 104-108 (2009).

41. A. J. Ridley, H. F. Paterson, C. L. Johnston, D. Diekmann, A. Hall, The small GTP-binding protein rac regulates growth factor-induced membrane ruffling. Cell 70, 401-410 (1992).

42. E. Kardash et al., A role for Rho GTPases and cell-cell adhesion in single-cell motility in vivo. Nat. Cell Biol. 12, 47-53, 1-11 (2010).

43. M. J. Dayel, E. F. Y. Hom, A. S. Verkman, Diffusion of green fluorescent protein in the aqueous-phase lumen of endoplasmic reticulum. Biophys. J. 76, 2843-2851 (1999)

44. A. Boquet-Pujadas et al., BioFlow: A non-invasive, image-based method to measure speed, pressure and forces inside living cells. Sci. Rep. 7, 9178 (2017)

45. S. N. Hird, J. G. White, Cortical and cytoplasmic flow polarity in early embryonic cells of Caenorhabditis elegans. J. Cell Biol. 121, 1343-1355 (1993).

46. T. Y.-C. Tsai et al., Efficient front-rear coupling in neutrophil chemotaxis by dynamic myosin II localization. Dev. Cell 49, 189-205.e6 (2019).

47. T. Leung, X.-Q. Chen, E. Manser, L. Lim, The p160 RhoA-binding kinase ROK alpha is a member of a kinase family and is involved in the reorganization of the cytoskeleton. Mol. Cell. Biol. 16, 5313-5327 (1996).

48. V. Ruprecht et al., Cortical contractility triggers a stochastic switch to fast amoeboid cell motility. Cell 160, 673-685 (2015).

49. Y.-J. Liu, Confinement and low adhesion induce fast amoeboid migration of slow mesenchymal cells. Cell 160, 659-672 (2015).

50. R. Viswanatha, J. Wayt, P. Y. Ohouo, M. B. Smolka, A. Bretscher, Interactome analysis reveals ezrin can adopt multiple conformational states. J. Biol. Chem. 288, 35437-35451 (2013)

51. A. Bisaria, A. Hayer, D. Garbett, D. Cohen, T. Meyer, Membrane-proximal F-actin restricts local membrane protrusions and directs cell migration. Science 368, 1205-1210 (2020).

52. J. Carretero-Ortega et al., Phosphatidylinositol 3,4,5-triphosphate-dependent Rac exchanger 1 (P-Rex-1), a guanine nucleotide exchange factor for Rac, mediates an giogenic responses to stromal cell-derived factor-1/chemokine stromal cell derived factor-1 (SDF-1/CXCL-12) linked to Rac activation, endothelial cell migration, and in vitro angiogenesis. Mol. Pharmacol. 77, 435-442 (2010).

53. H. Li et al., Association between Goi2 and ELMO1/Dock180 connects chemokine signalling with Rac activation and metastasis. Nat. Commun. 4, 1706 (2013).

54. J. Vázquez-Prado, I. Bracho-Valdés, R. D. Cervantes-Villagrana, G. Reyes-Cruz, G $\beta \gamma$ pathways in cell polarity and migration linked to oncogenic GPCR signaling: Potentia relevance in tumor microenvironment. Mol. Pharmacol. 90, 573-586 (2016).

55. X. Li, Y. Miao, D. S. Pal, P. N. Devreotes, Excitable networks controlling cell migration during development and disease. Semin. Cell Dev. Biol. 100, 133-142 (2019).

56. A. Van Keymeulen et al., To stabilize neutrophil polarity, PIP3 and Cdc42 augment RhoA activity at the back as well as signals at the front. J. Cell Biol. 174, 437-445 (2006).

57. E. Zatulovskiy, R. Tyson, T. Bretschneider, R. R. Kay, Bleb-driven chemotaxis of Dic tyostelium cells. J. Cell Biol. 204, 1027-1044 (2014).

58. K. Tsujita, T. Takenawa, T. Itoh, Feedback regulation between plasma membrane tension and membrane-bending proteins organizes cell polarity during leading edge formation. Nat. Cell Biol. 17, 749-758 (2015).

59. B. Rouven Brückner, A. Pietuch, S. Nehls, J. Rother, A. Janshoff, Ezrin is a major regulator of membrane tension in epithelial cells. Sci. Rep. 5, 14700 (2015).

60. A. Diz-Muñoz et al., Membrane tension acts through PLD2 and mTORC2 to limit actin network assembly during neutrophil migration. PLoS Biol. 14, e1002474 (2016)

61. S. Gross-Thebing et al., Using migrating cells as probes to illuminate features in live embryonic tissues. Sci. Adv. 6, eabc5546.

62. M. Krieg et al., Tensile forces govern germ-layer organization in zebrafish. Nat. Cell Biol. 10, 429-436 (2008).

63. P. Aleström et al., Zebrafish: Housing and husbandry recommendations. Lab. Anim. 54, 213-224.

64. J. Hartwig et al., Temporal control over the initiation of cell motility by a regulator of G-protein signaling. Proc. Natl. Acad. Sci. U.S.A. 111, 11389-11394 (2014)

65. M. Köprunner, C. Thisse, B. Thisse, E. Raz, A zebrafish nanos-related gene is essential for the development of primordial germ cells. Genes Dev. 15, 2877-2885 (2001)

66. F. de Chaumont et al., Icy: An open bioimage informatics platform for extended reproducible research. Nat. Methods 9, 690-696 (2012). 